\title{
Love Is All You Need: User Preferences of Strategies for Mediating Intimate Relationships Through Technology
}

\author{
Yulia Silina \\ Queen Mary University of London \\ Mile End Rd, London E1 4NS, UK \\ CuriousYulia@gmail.com
}

\author{
Adela Apetroaia \\ Newham College University Centre \\ Welfare Road, London E15 4HT \\ Adela.Apetroaia@newham.ac.uk
}

\author{
Anne Hsu \\ Queen Mary University of London \\ Mile End Rd, London E1 4NS, UK \\ Anne.Hsu@qmul.ac.uk
}

\begin{abstract}
Maintaining a sense of relatedness with loved-ones is one of the top human needs and predictors of wellbeing. $\mathrm{HCl}$ research has increasingly focused on the ways and implications of mediating relatedness through technology. However, current research is at times removed from established theories in related disciplines and it lacks consistent tools for selecting user groups and appropriate interaction strategies. Our paper identifies the characteristics of potential users of tech-mediated devices in conjunction with related disciplines. It then examines users' preferences for the six Strategies of Mediating Intimate Relationships though Technology, identified by Hassenzahl et al. in the paper "All you need is Love", in interaction within different types of relationships (with friends, partners, siblings, etc.). Our paper proposes expanding these strategies to account for varied levels of reciprocity and for essential/unwanted strategies identified by users in different types of relationships. It concludes with implications and suggestions for future research.
\end{abstract}

Computer-mediated relationships, attachment, relatedness, secure base, experience design, positive computing.

\section{INTRODUCTION}

\subsection{Computer-Mediated Intimate Relationships}

It is widely agreed that "love, and general feeling of being related to significant others are crucial to people's life satisfaction and happiness" (Hassenzahl at al., 2012), and that maintaining the sense of relatedness (also known in $\mathrm{HCl}$ as connectedness, intimacy, love, belonging, closeness, or togetherness) is one of the top human needs (Sheldon et al., 2001). There are many means of communication that help loved-ones to stay in touch, from analogue keepsakes and letters to video-conferencing and complex social networks (Wright \& Webb, 2010). Among these, there is a subset of augmented keepsake-like artefacts that enhance the sense of relatedness to loved-ones (LO) and maintain their psychological closeness.

Hassenzahl et al. reviewed and consolidated prior research within $\mathrm{HCl}$ on Computer-Mediated Intimate Relationships (Hassenzahl at al., 2012). Authors pointed out gaps in the knowledge, highlighted inconsistencies in tools and methodologies, and the lack of incorporation of established research from other related disciplines. Summarizing the approaches described in previous $\mathrm{HCl}$ papers, the authors identified six reciprocal strategies employed by researchers for designing artefact that mediate intimate relationships through technology (Figure 1).
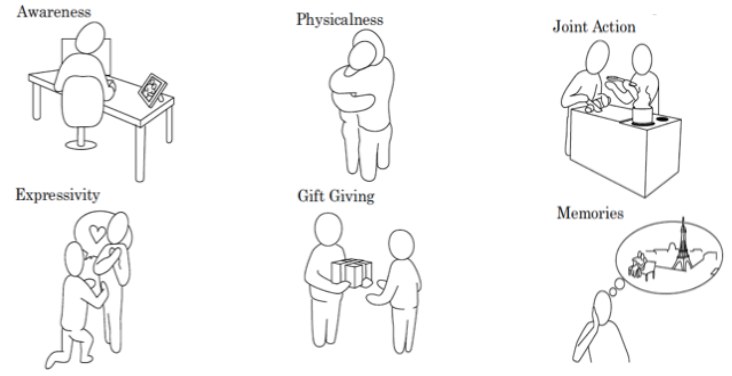

Figure 1: Current Strategies for Mediation of Intimate Relationships Through Tech. (Hassenzahl at al., 2012)

\subsection{Theory of Intimate Relationships}

Attachment Theory is the core motivational and behavioural system that can explain someone's intimate relationships (Hazan \& Shaver, 1994). It is used as a base for understanding communication between relational figures (including communication via analogue and technological artefacts).

Relational figures vary, but are predominantly represented by parents, offsprings, grandparents, grandchildren, siblings, best friends and romantic partners (Table 1). 
Table 1: List of common Relational Figures (loved-ones)

\begin{tabular}{|l|l|l|}
\hline Relationship & \multicolumn{1}{|c|}{ Female } & \multicolumn{1}{c|}{ Male } \\
\hline Partner & Girlfriend/Wife & Boyfriend/Husb \\
\hline Friend & Female Friend & Male Friend \\
\hline Sibling & Sister & Brother \\
\hline Parent & Mother figure & Father figure \\
\hline Offspring & Daughter & Son \\
\hline Grandparent & Grandmother & Grandfather \\
\hline Grandchild & Granddaughter & Grandson \\
\hline Other & Other $F$ & Other $M$ \\
\hline
\end{tabular}

Attachment is defined as the ability to use one's relational attachment figure as a secure base from which to explore the world. In this context, perceived proximity and availability of ones loved-ones lead to increased wellbeing and living a bigger, richer life (Feeney \& Collins, 2014).

Over $60 \%$ of people can be characterized as securely attached (Mickelson et al., 1997), which means that they are comfortable depending on others and having others depend on them. The other two main attachment styles are: avoidant or dismissive and anxious-ambivalent or preoccupied (Bartholomew, 1990).

Although the relationships are dynamic (Knapp, \& Daly, 2011), generalized attachment styles tend to be consistent over one's lifespan (Fraley, 2002). However, the specific manifestation of these attachment styles could be different for different relational figures (Hazan \& Shaver, 1994). For example, someone could have the generalized tendency to trust romantic partners, thus having a secure attachment style towards them, but they may have an avoidant relationship with one of parents.

\subsection{Motivation}

From the secure base perspective, the role of a successful artefact that enables tech-mediated communication is to increase the perceived availability of the loved-ones and to reduce anxiety and avoidance using relevant mediation strategies. Although the strategies identified by Hassenzahl et al. were instrumental in establishing a common ground in the field, it focused on the strategies used by researchers and designers. To date, we are not aware of any work that has been done to examine these strategies from the perspective of users of tech-mediated artefacts. In this paper we intend to fill this gap, starting from identifying the characteristics of the users of such artefacts.

In view of the Attachment Theory, in design of the systems for mediating intimate relationships, it is not only important to consider the immediate user, but also a type of user-pair (dyad). Several studies, examined by Hassenzahl et al. and some newer studies that followed since involved friends and family members. However, most studies focused on romantic partners, often in long-distance relationships (LDR). Despite being specific about types of dyads, these studies do not investigate or discuss differences in preferences of these strategies among different dyad types.

In the context of intrapersonal communication, expectations of communication, as well as the levels and willingness of self-disclosure affect the dynamics of communication between people (Knapp, \& Daly, 2011). Similarly, receiving (From) and sending (To) communication are distinctly different actions with different motivational triggers and potential outcomes. Thus, strategies could be described as shown in Table 2.

Table 2: Directions of Mediation Strategies

\begin{tabular}{|l|l|l|l|}
\hline \multicolumn{1}{|c|}{ Strategy } & \multicolumn{3}{c|}{ Possible Direction of Strategy } \\
\hline Awareness & Reciprocal & From LO & To LO \\
\hline Expressivity & Reciprocal & From LO & To LO \\
\hline Physicalness & Reciprocal & From LO & To LO \\
\hline Gift Giving & Reciprocal & From LO & To LO \\
\hline Memories & Reciprocal & From LO & To LO \\
\hline Joint Action & Reciprocal & n/a & $n / a$ \\
\hline
\end{tabular}

The strategies that might appear to be reciprocal may not be equally desired by both parties within specific types of relationships. However, neither research, examined by Hassenzahl et al., nor work that came out since consider possible differences in the preferences for asymmetrical engagement.

Furthermore, consistent with theories within Relationship and Communication Psychology (Duck, 2007), it is reasonable to assume that different mediation strategies will have different levels of importance for different relational figures and will depending on the quality of relationship and frequency of seeing each other. For example, sharing Memories might be equally preferred by all relational dyads, but Physicalness might be preferred more by romantic partners than by friends or siblings who might prefer Joint Action instead.

\section{RESEARCH AIM}

There are two main aims of this research. The first aim is to understand the characteristics of the people that would like to engage with augmented artefacts for mediation of their relationships (users). The second, is to examine how these users prefer to engage with the variety of their loved-ones through these artefacts, using the current set of six mediation strategies (Hassenzahl at al., 2012) (Figure1) as a base. As such, we intend to explore following research questions: 


\subsection{Questions: Users Characteristics}

Q1: What relational figures do people miss the most when they are apart?

Q2: Do types of relational dyads, gender of relational figures, quality of the relationship, or frequency of seeing relational figure affect the desire to using augmented keepsake artefacts to stay in touch with relational figures?

\subsection{Questions: Preferences of Mediation Strategies}

Q3: Are mediation strategies inherently reciprocal? Or are they directional? For example: users who want to be Aware of their loved-ones, might not want to disclose the same information about themselves.

Q4: Are all mediation strategies equally essential? Or are there are some strategies that users find more essential than others, or perhaps do not want at all?

Q5: Do all types of dyads equally prefer the same types of strategies? Or do people in different types relationships, such as with romantic partners, friends and different members of the family prefer their relationships mediated through different combinations of strategies? For example: Physicalness might be most important strategy for romantic partners, whereas Awareness for might be for offsprings.

\section{STUDY}

\subsection{Method}

To unpack our research aims, we deployed a semistructured online questionnaire to participants, interested in keeping in touch with their loved-ones. The questionnaire was designed to obtain insights into the characteristics of potential users of augmented artefacts for mediation of relationship with common relational figures and their preferences for mediation strategies. We rephrased original descriptions (Hassenzahl at al., 2012) to remove technical terms and make them accessible to participants (Table 3 ).

To account for potential differences in the preference for asymmetrical engagement, we gave To and From directions to six current mediation strategies, described by Hassenzahl et al. Using logic-based platform, participants were asked if they had a particular relational figure, for example: Do you have a sister? Yes / No. If they answered Yes and had more than one, they were asked to pick their favourite and invited to do following: "Imagine, you have a very special object that lets two of you to keep in touch without having to call or write by using following scenarios:", followed by the directional descriptions of Mediation Strategies (Table 3).
Table 3: Revised Description of Mediation Strategies.

\begin{tabular}{|c|c|}
\hline Strategy & Description \\
\hline $\begin{array}{l}\text { Awareness } \\
\text { (To) }\end{array}$ & $\begin{array}{l}\text { Get a constant feeling of your loved- } \\
\text { one's presence, through knowing her } \\
\text { moods, activities, whereabouts, } \\
\text { environment (weather, sounds), etc.. }\end{array}$ \\
\hline $\begin{array}{l}\text { Awareness } \\
\text { (From) }\end{array}$ & $\begin{array}{l}\text { Continuously share your moods, } \\
\text { whereabouts, activities and } \\
\text { environment so that loved-one might } \\
\text { feel your presence? }\end{array}$ \\
\hline $\begin{array}{l}\text { Expressivit } \\
y \\
\text { (To) }\end{array}$ & $\begin{array}{l}\text { View or feel a secret coded message } \\
\text { when your loved-one lets you know } \\
\text { that she is thinking of you, that she is } \\
\text { happy, that she misses you, etc... }\end{array}$ \\
\hline $\begin{array}{l}\text { Expressivit } \\
y \\
\text { (From) }\end{array}$ & $\begin{array}{l}\text { Discreetly send a secret coded } \\
\text { message to let your loved-one know } \\
\text { you are thinking of her, that you are } \\
\text { happy, that you miss her, etc... }\end{array}$ \\
\hline $\begin{array}{l}\text { Physicalne } \\
\text { ss } \\
\text { (To) }\end{array}$ & $\begin{array}{l}\text { Experience your loved-one's physical } \\
\text { gestures, like hugs, gentle strokes, } \\
\text { etc... or her physical state, like body } \\
\text { heat, heartbeat, etc... }\end{array}$ \\
\hline $\begin{array}{l}\text { Physicalne } \\
\text { ss } \\
\text { (From) }\end{array}$ & $\begin{array}{l}\text { Transmit your physical gestures or } \\
\text { physical state to your loved-one. }\end{array}$ \\
\hline $\begin{array}{l}\text { Gift Giving } \\
\text { (To) }\end{array}$ & $\begin{array}{l}\text { Receive a gift from your loved-one, } \\
\text { such as a song, a photograph, } \\
\text { directions to some special pace, etc.. }\end{array}$ \\
\hline $\begin{array}{l}\text { Gift Giving } \\
\text { (From) }\end{array}$ & Send a gift to your loved-one \\
\hline $\begin{array}{l}\text { Memories } \\
\text { (To) }\end{array}$ & $\begin{array}{l}\text { Revisit memories of special moments } \\
\text { of you and your loved-one through } \\
\text { sounds, smells, images, etc... }\end{array}$ \\
\hline $\begin{array}{l}\text { Memories } \\
\text { (From) }\end{array}$ & $\begin{array}{l}\text { Record and keep new special moments } \\
\text { of you and your loved-one. }\end{array}$ \\
\hline $\begin{array}{l}\text { Joint Action } \\
\text { (To \& } \\
\text { From) }\end{array}$ & $\begin{array}{l}\text { Do things together with your loved-one } \\
\text { over distance, like play a game, listen } \\
\text { to the same song, etc... }\end{array}$ \\
\hline
\end{tabular}

Participants were then asked to rate these scenarios on a 4-point Likert scale ranging from "essential", to "I would not want this". Following this, participants were asked if they were interested in having such object for communication with this person; and if so, what this object might be. Participants were also asked to rate the quality of the relationship they had with this person on a scale from "Love very much" (10) to "Neutral" (0) to "Not good" (-2 or less). Finally, they were asked to indicate how frequently they usually see this person on a 5-point Likert scale from "Daily" to "Every few years".

The set of questions repeated for each category of relational figures in Table 1 . The questionnaire concluded by asking whom participants missed the most when apart, and demographic questions about participant's age and gender. Participants were given an opportunity to also leave an open comment about any aspects related to this study. 


\subsection{Results: Participants}

We received complete responses from 147 participants (63 of whom identified themselves as male, 81 as female and 3 as other). Due to the low count of other, we removed them from further calculations, leaving us with 144 participants. The median age of the participants was 32.5 (20 min and 85 max and five outliers: 58, 59, 61, 65, 85 which we retained as we did not looking at the age as a factor in mediation of relationships.

\subsection{Results: Relational Dyads}

\subsubsection{Reported types of relational figures}

Overall, participants reported 609 types of relational figures (260 males, 377 females and 2 other) (Figure 2) with median of 5 types per participant ( 1 min, 10 max and no outliers). In addition to common relational figures (Table 1), under other (3) participants reported niece, god and pet.

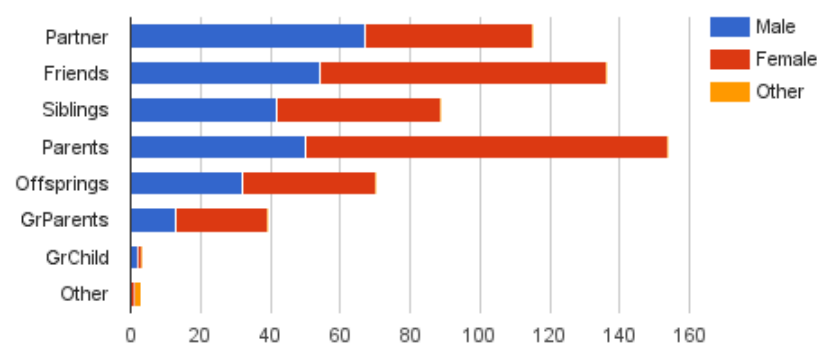

Figure 2: Reported types of relational figures

\section{Q1: Reported types of most-missed loved-ones}

Only a quarter (114) of all reported relational figures were most-missed. Partners were by far the most prominent most-missed group (88), followed by offsprings (18), parents (14) and friends (11) (Fig 3).

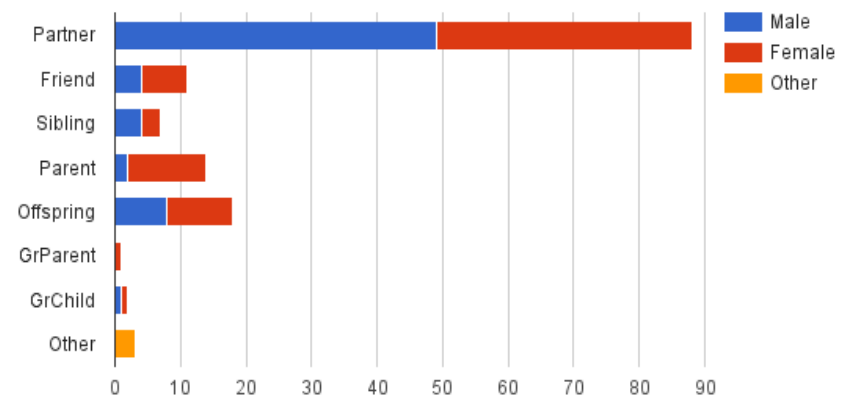

Figure 3: Most-missed relational figures

There was a significant difference between reported and most-missed relational figures. $\left(\mathrm{X}^{2}(1\right.$, $\mathrm{N}=702)=108.9143$; $\mathrm{p}$-value $=0.00001<.05$ ) .

Information provided for four of the relational figures was incomplete, and these were removed from further analysis, as were grandchildren (3) and others (3) categories whose counts were less than 6 , required for chi-square statistic. Resulting data was arranged by gender into dyads, with participants (potential users) followed by potential loved-ones (Table 4).

Table 4: Reported types of relational dyads by gender

\begin{tabular}{|l|l|l|l|l|l|}
\hline User - LO & $\mathbf{M - M}$ & $\mathbf{M}-\mathbf{F}$ & $\mathbf{F - M}$ & $\mathbf{F - F}$ & Total \\
\hline Partner - & 2 & 44 & 64 & 2 & $\mathbf{1 1 2}$ \\
Partner & $2 \%$ & $39 \%$ & $57 \%$ & $2 \%$ & $100 \%$ \\
\hline Friend - & 37 & 25 & 17 & 57 & $\mathbf{1 3 6}$ \\
Friend & $27 \%$ & $18 \%$ & $13 \%$ & $42 \%$ & $100 \%$ \\
\hline Sibling - & 13 & 16 & 29 & 31 & $\mathbf{8 9}$ \\
Sibling & $15 \%$ & $18 \%$ & $33 \%$ & $35 \%$ & $100 \%$ \\
\hline Parent - & 10 & 9 & 23 & 28 & $\mathbf{7 0}$ \\
Offspring & $14 \%$ & $13 \%$ & $33 \%$ & $40 \%$ & $100 \%$ \\
\hline Offspring - & 21 & 43 & 29 & 60 & $\mathbf{1 5 3}$ \\
Parent & $14 \%$ & $28 \%$ & $19 \%$ & $39 \%$ & $100 \%$ \\
\hline Grandchild - & 6 & $\mathbf{9}$ & 7 & $\mathbf{1 7}$ & $\mathbf{3 9}$ \\
Grandparent & $15 \%$ & $23 \%$ & $18 \%$ & $44 \%$ & $100 \%$ \\
\hline Total & $\mathbf{8 9}$ & $\mathbf{1 4 6}$ & $\mathbf{1 6 9}$ & $\mathbf{1 9 5}$ & $\mathbf{5 9 9}$ \\
& $15 \%$ & $24 \%$ & $28 \%$ & $33 \%$ & $100 \%$ \\
\hline
\end{tabular}

\subsubsection{Quality of relationships}

Participants were asked to rate their relationship with a person in each relational dyad from 10 ("Love very much") through 0 ("Neutral") to -2 and below ("Not good"). Responses were aggregated into 3 categories Loving (7 to 10), Good (3 to 6) and Poor (below 3). Over $70 \%$ (428) of relationships were Loving, 9\% (55) of relational dyads were reported to be Poor, and remainder (116) Good (Figure 4).

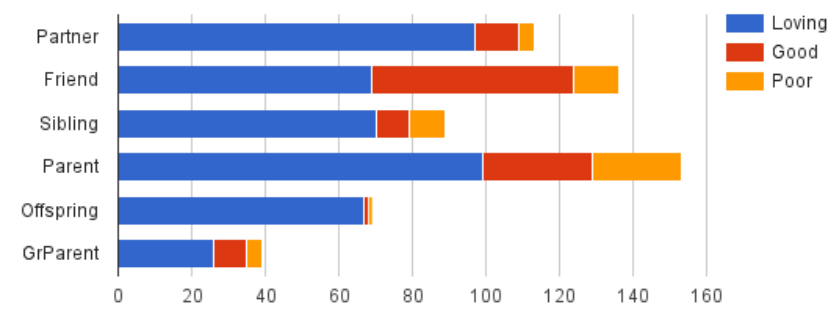

Figure 4: Quality of relationship with relational figures

\subsubsection{Frequency of Seeing Loved-ones}

Participants were asked to rate the frequency of seeing a person within the relational dyad from 1 (Every few years) to 5 (Daily). The biggest group (about 34\%, 204) of dyads reported to see each other "Daily", followed by "Few times a year" (130), "Few times a month" (109), "Few times a week" (80) and "Every few years" (76) (Figure 5).

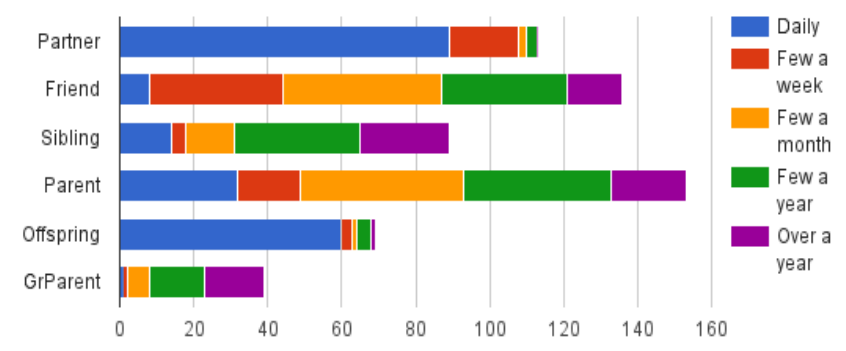

Figure 5:. Frequency of seeing relational figures 


\subsection{Results: User Characteristics}

To determine who the users of the mediated artefacts might be, participants were asked whether they want or do not want to have a special keepsake object that allowed them to keep in touch with a particular person that they reported without having to call or write. Resulted data was aggregated by dyad characteristics (Table 5 ).

Table 5: User Characteristics for desire to have keepsake

\begin{tabular}{|c|c|c|c|c|}
\hline \multicolumn{2}{|c|}{ Characteristics } & Want & Not & Total \\
\hline \multirow{6}{*}{ 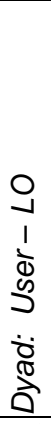 } & Partner - Partner & $\begin{array}{l}111 \\
99 \%\end{array}$ & $\begin{array}{l}1 \\
1 \%\end{array}$ & $\begin{array}{l}112 \\
100 \%\end{array}$ \\
\hline & Friend - Friend & $\begin{array}{l}103 \\
76 \%\end{array}$ & $\begin{array}{l}33 \\
24 \%\end{array}$ & $\begin{array}{l}136 \\
100 \%\end{array}$ \\
\hline & Sibling - Sibling & $\begin{array}{l}66 \\
74 \%\end{array}$ & $\begin{array}{l}23 \\
26 \%\end{array}$ & $\begin{array}{l}89 \\
100 \%\end{array}$ \\
\hline & Parent - Offspring & $\begin{array}{l}118 \\
77 \%\end{array}$ & $\begin{array}{l}35 \\
23 \%\end{array}$ & $\begin{array}{l}153 \\
100 \%\end{array}$ \\
\hline & Offspring - Parent & $\begin{array}{l}67 \\
96 \%\end{array}$ & $\begin{array}{l}3 \\
4 \%\end{array}$ & $\begin{array}{l}70 \\
100 \%\end{array}$ \\
\hline & GrChld- GrParent & $\begin{array}{l}28 \\
72 \%\end{array}$ & $\begin{array}{l}11 \\
28 \%\end{array}$ & $\begin{array}{l}39 \\
100 \% \\
\end{array}$ \\
\hline \multicolumn{2}{|c|}{ TOTAL } & $\begin{array}{l}493 \\
82 \% \\
\end{array}$ & $\begin{array}{l}106 \\
18 \%\end{array}$ & $\begin{array}{l}599 \\
100 \% \\
\end{array}$ \\
\hline \multirow{4}{*}{$\begin{array}{l}1 \\
\frac{1}{d} \\
\frac{d}{0} \\
\frac{d}{0} \\
0\end{array}$} & Female - Female & $\begin{array}{l}162 \\
83 \%\end{array}$ & $\begin{array}{l}33 \\
17 \%\end{array}$ & $\begin{array}{l}195 \\
100 \%\end{array}$ \\
\hline & Female - Male & $\begin{array}{l}146 \\
86 \%\end{array}$ & $\begin{array}{l}23 \\
14 \%\end{array}$ & $\begin{array}{l}\mathbf{1 6 9} \\
100 \%\end{array}$ \\
\hline & Male - Female & $\begin{array}{l}124 \\
15 \%\end{array}$ & $\begin{array}{l}22 \\
15 \%\end{array}$ & $\begin{array}{l}\mathbf{1 4 6} \\
100 \%\end{array}$ \\
\hline & Male - Male & $\begin{array}{l}61 \\
31 \%\end{array}$ & $\begin{array}{l}2831 \\
\%\end{array}$ & $\begin{array}{l}\mathbf{8 9} \\
100 \%\end{array}$ \\
\hline \multicolumn{2}{|c|}{ TOTAL } & $\begin{array}{l}493 \\
82 \%\end{array}$ & $\begin{array}{l}106 \\
18 \%\end{array}$ & $\begin{array}{l}599 \\
100 \%\end{array}$ \\
\hline \multirow{3}{*}{ 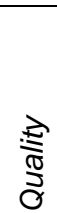 } & Loving (7-10) & $\begin{array}{l}393 \\
92 \%\end{array}$ & $\begin{array}{l}35 \\
8 \%\end{array}$ & $\begin{array}{l}\mathbf{4 2 8} \\
100 \%\end{array}$ \\
\hline & Good (3-6) & $\begin{array}{l}76 \\
66 \%\end{array}$ & $\begin{array}{l}40 \\
34 \%\end{array}$ & $\begin{array}{l}116 \\
100 \%\end{array}$ \\
\hline & Poor (2 \& below) & $\begin{array}{l}24 \\
44 \%\end{array}$ & $\begin{array}{l}31 \\
56 \%\end{array}$ & $\begin{array}{l}55 \\
100 \%\end{array}$ \\
\hline \multicolumn{2}{|c|}{ TOTAL } & $\begin{array}{l}493 \\
82 \% \\
\end{array}$ & $\begin{array}{l}106 \\
18 \% \\
\end{array}$ & $\begin{array}{l}599 \\
100 \% \\
\end{array}$ \\
\hline \multirow{5}{*}{$\begin{array}{l}\text { dे } \\
\frac{d}{d} \\
\frac{\sigma}{d} \\
\frac{d}{1}\end{array}$} & 5 - Daily & $\begin{array}{l}193 \\
95 \%\end{array}$ & $\begin{array}{l}11 \\
5 \%\end{array}$ & $\begin{array}{l}\mathbf{2 0 4} \\
100 \%\end{array}$ \\
\hline & 4 - Few a week & $\begin{array}{l}72 \\
90 \%\end{array}$ & $\begin{array}{l}8 \\
10 \%\end{array}$ & $\begin{array}{l}80 \\
100 \%\end{array}$ \\
\hline & 3 - Few a month & $\begin{array}{l}81 \\
74 \%\end{array}$ & $\begin{array}{l}28 \\
26 \%\end{array}$ & $\begin{array}{l}\mathbf{1 0 9} \\
100 \%\end{array}$ \\
\hline & 2 - Few a year & $\begin{array}{l}98 \\
75 \%\end{array}$ & $\begin{array}{l}32 \\
25 \%\end{array}$ & $\begin{array}{l}\mathbf{1 3 0} \\
100 \%\end{array}$ \\
\hline & $\begin{array}{l}1-\text { Every few } \\
\text { years }\end{array}$ & $\begin{array}{l}49 \\
64 \%\end{array}$ & $\begin{array}{l}27 \\
36 \%\end{array}$ & $\begin{array}{l}76 \\
100 \%\end{array}$ \\
\hline \multicolumn{2}{|c|}{ TOTAL } & $\begin{array}{l}493 \\
82 \%\end{array}$ & $\begin{array}{l}106 \\
18 \%\end{array}$ & $\begin{array}{l}599 \\
100 \%\end{array}$ \\
\hline
\end{tabular}

Q2a: Desire by Type of Relational Dyad

Participants wanted to use an augmented keepsake for interaction with $82 \%$ (493) of relational figures (Figure 6). There was no significant difference between individual types of dyads, but partners and parents were more likely to want an augmented keepsake than the rest of the groups $\left(x^{2}(1, N=599)=43.12 ; p\right.$-value $=$ $0.00001<.05)$

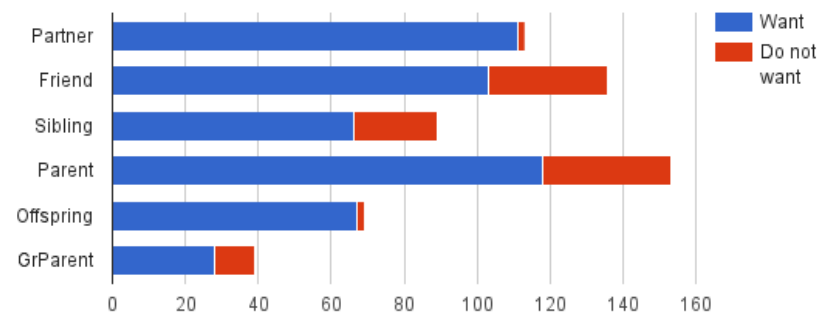

Figure 6: Desire to have keepsakes by relational figures

\section{Q2b: Desire by Gender}

Overall, there was no significant difference between the preferences to use the keepsakes based on the gender of the participants. Similarly, when looking at relational dyads, there was no significant difference between pairs that have at least one female. However, reported male-male dyads were significantly less inclined to want keepsakes than all others $\left(x^{2}(1, N=599)=13.60\right.$; $p$-value $=0.000226<.05)$.

\section{Q2c: Desire by Quality of Relationships}

The chi-square test indicates that quality of relationship influences the desire to use augmented keepsake for communion with a relational figure $\left(x^{2}\right.$ $(1, N=599)=105.53 ; p$-value $<0.00001<.05)$, with better relationship making keepsakes more desired.

\section{Q2d: Desire by Frequency of Seeing Relational Figure}

When comparing desire for the keepsakes based on the frequency of seeing each other, there was no significant difference between individual groups of people who see their relational figure "Few times a month" or less (groups 1, 2 and 3). Similarly, there was no significant differences between groups of people who see their loved-one "Few times a week" or more often (groups 4 and 5). However, people who see their loved-ones "Few times a week" or more often, were more likely to want a keepsake than people who see each other less than "Few times a month" $\left(X^{2}(1, N=599)=\right.$ 44.92; $p$-value $<0.00001<.05)$.

\subsection{Results: Preferences of Mediation Strategies}

To get a clear user perspective on Mediation Strategies, we removed from further analysis all relational dyads in which participants did not want to have the augmented keepsakes, leaving us with 493 relational dyads (Table 6 ).

Participants were asked to imagine that they had a very special object that lets them keep in touch with a particular loved-one through the use of scenarios in Table 3. They were then asked to rank the 
Table 6: Reported types of relational dyads by gender

\begin{tabular}{|l|l|l|l|l|l|}
\hline User - LO & M-M & M-F & F-M & F-F & Total \\
\hline Partner - & 2 & 42 & 65 & 2 & $\mathbf{1 1 1}$ \\
Partner & $2 \%$ & $38 \%$ & $59 \%$ & $2 \%$ & $100 \%$ \\
\hline Friend - & 24 & 20 & 13 & 46 & $\mathbf{1 0 3}$ \\
Friend & $23 \%$ & $19 \%$ & $13 \%$ & $45 \%$ & $100 \%$ \\
\hline Sibling - & 11 & 11 & 20 & 24 & $\mathbf{6 6}$ \\
Sibling & $17 \%$ & $17 \%$ & $30 \%$ & $36 \%$ & $100 \%$ \\
\hline Parent- & 8 & 9 & 22 & 28 & $\mathbf{6 7}$ \\
Offspring & $12 \%$ & $13 \%$ & $33 \%$ & $42 \%$ & $100 \%$ \\
\hline Offspring- & 14 & 35 & 22 & 47 & $\mathbf{1 1 8}$ \\
Parent & $12 \%$ & $30 \%$ & $19 \%$ & $40 \%$ & $100 \%$ \\
\hline GrChld- & 2 & 7 & 4 & 15 & $\mathbf{2 8}$ \\
GrParent & $7 \%$ & $25 \%$ & $14 \%$ & $54 \%$ & $100 \%$ \\
\hline Total & $\mathbf{6 1}$ & $\mathbf{1 2 4}$ & $\mathbf{1 4 6}$ & $\mathbf{1 6 2}$ & $\mathbf{4 9 3}$ \\
& $12 \%$ & $25 \%$ & $30 \%$ & $33 \%$ & $100 \%$ \\
\hline
\end{tabular}

importance of these scenarios from I would not want this, Ambivalent and Essential. All instances of 2way From and 2-way To that occurred simultaneously within the same dyad (Table 7) were labelled as "Reciprocal" and given a single score.

\section{Q3: Reciprocity of Mediation Strategies}

Most current studies of mediation of intimate relationships use reciprocal strategies. Both Figures 7 and 9 clearly illustrate the importance of reciprocity. However, when looking at mediation strategies (excluding inherently reciprocal Joint Action), the difference between reciprocal and onedirectional (non-reciprocal) strategies for both essential and unwanted cases was significant. (Essential: $x^{2}=12.06$; $p$-value < $0.017<.05$; Unwanted: $\mathrm{x}^{2}=30.36$; $\mathrm{p}$-value $<0.00001<.05$ ).

\section{Q4a: Essential Mediation Strategies.}

Current studies on mediation of intimate relationships do not address the differences in importance of the strategies, suggesting that the strategies are equally wanted within all relational dyads. Using this as an underling assumption, for 493 dyads, we could anticipate 989 instances for each of the six original strategies (493 for From, 493 for To directions) with $100 \%$ essential. Instead, when looking at the total ranking of all strategies, only about $33 \%(1,966)$ of all possible instances of From and To were identified by participants as essential (Figure 7).

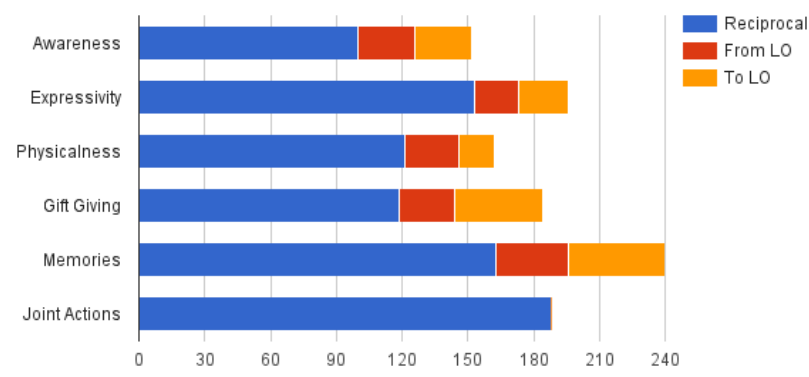

Figure 7: Essential Mediation Strategies scores
Table 7: Summary of reported From \& To Strategies

\begin{tabular}{|c|c|c|c|c|c|c|}
\hline \multicolumn{3}{|c|}{$\begin{array}{c}\text { Reported } \\
\text { Strategy }\end{array}$} & $\begin{array}{l}\text { Esse } \\
\text { ntial }\end{array}$ & $\begin{array}{c}\text { Ambiv } \\
\text { alent. }\end{array}$ & $\begin{array}{c}\text { Not } \\
\text { wantd }\end{array}$ & Total \\
\hline \multirow{4}{*}{ 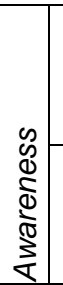 } & & From & $263 \%$ & $384 \%$ & $91 \%$ & $\begin{array}{l}73 \\
7 \%\end{array}$ \\
\hline & ָे & To & $263 \%$ & $303 \%$ & $172 \%$ & $\begin{array}{c}73 \\
7 \%\end{array}$ \\
\hline & & From & $\begin{array}{l}100 \\
10 \%\end{array}$ & $\begin{array}{l}211 \\
21 \%\end{array}$ & $\begin{array}{l}109 \\
11 \%\end{array}$ & $\begin{array}{l}420 \\
43 \%\end{array}$ \\
\hline & $\mid \begin{array}{l}\text { ते } \\
\text { సे }\end{array}$ & To & $\begin{array}{l}100 \\
10 \% \\
\end{array}$ & $\begin{array}{l}211 \\
21 \% \\
\end{array}$ & $\begin{array}{l}109 \\
11 \% \\
\end{array}$ & $\begin{array}{l}420 \\
43 \% \\
\end{array}$ \\
\hline \multicolumn{3}{|c|}{ TOTAL } & $\begin{array}{l}252 \\
26 \%\end{array}$ & $\begin{array}{l}490 \\
50 \%\end{array}$ & $\begin{array}{l}244 \\
25 \%\end{array}$ & $\begin{array}{l}986 \\
100 \%\end{array}$ \\
\hline \multirow{4}{*}{ 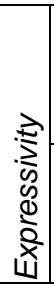 } & & From & $\begin{array}{l}20 \\
2 \% \\
\end{array}$ & $\begin{array}{l}30 \\
3 \%\end{array}$ & $\begin{array}{l}8 \\
1 \% \\
\end{array}$ & $\begin{array}{l}58 \\
6 \% \\
\end{array}$ \\
\hline & ते & To & $\begin{array}{l}23 \\
2 \%\end{array}$ & $\begin{array}{l}28 \\
3 \% \\
\end{array}$ & $\begin{array}{l}7 \\
1 \% \\
\end{array}$ & $\begin{array}{l}58 \\
6 \% \\
\end{array}$ \\
\hline & & From & $\begin{array}{l}153 \\
16 \%\end{array}$ & $\begin{array}{l}216 \\
22 \%\end{array}$ & $\begin{array}{l}66 \\
7 \%\end{array}$ & $\begin{array}{l}\mathbf{4 3 5} \\
44 \%\end{array}$ \\
\hline & 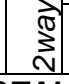 & To & $\begin{array}{l}153 \\
16 \% \\
\end{array}$ & $\begin{array}{l}216 \\
22 \% \\
\end{array}$ & $\begin{array}{l}66 \\
7 \% \\
\end{array}$ & $\begin{array}{l}\mathbf{4 3 5} \\
44 \% \\
\end{array}$ \\
\hline \multicolumn{3}{|c|}{ TOTAL } & $\begin{array}{l}349 \\
35 \%\end{array}$ & $\begin{array}{l}490 \\
50 \%\end{array}$ & $\begin{array}{l}147 \\
15 \% \\
\end{array}$ & $\begin{array}{l}986 \\
100 \%\end{array}$ \\
\hline \multirow{4}{*}{ 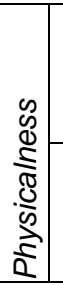 } & & From & $\begin{array}{l}25 \\
3 \% \\
\end{array}$ & $\begin{array}{l}26 \\
3 \% \\
\end{array}$ & $\begin{array}{c}14 \\
1 \% \\
\end{array}$ & $\begin{array}{l}65 \\
7 \% \\
\end{array}$ \\
\hline & 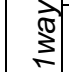 & To & $\begin{array}{l}16 \\
2 \%\end{array}$ & $\begin{array}{l}36 \\
4 \%\end{array}$ & $\begin{array}{l}13 \\
1 \%\end{array}$ & $\begin{array}{l}65 \\
7 \%\end{array}$ \\
\hline & & From & $\begin{array}{l}121 \\
12 \%\end{array}$ & $\begin{array}{l}210 \\
21 \%\end{array}$ & $\begin{array}{l}97 \\
10 \%\end{array}$ & $\begin{array}{l}\mathbf{4 2 8} \\
43 \%\end{array}$ \\
\hline & $\mid \begin{array}{l}\overrightarrow{\widetilde{\Omega}} \\
\text { సे }\end{array}$ & To & $\begin{array}{l}121 \\
12 \% \\
\end{array}$ & $\begin{array}{l}210 \\
21 \% \\
\end{array}$ & $\begin{array}{l}97 \\
10 \% \\
\end{array}$ & $\begin{array}{l}428 \\
43 \% \\
\end{array}$ \\
\hline \multicolumn{3}{|c|}{ TOTAL } & $\begin{array}{l}283 \\
29 \% \\
\end{array}$ & $\begin{array}{l}482 \\
49 \% \\
\end{array}$ & $\begin{array}{l}221 \\
22 \% \\
\end{array}$ & $\begin{array}{l}986 \\
100 \% \\
\end{array}$ \\
\hline \multirow{4}{*}{$\begin{array}{l}5 \\
5 \\
0 \\
0 \\
: 0\end{array}$} & & From & $253 \%$ & $384 \%$ & $162 \%$ & $\begin{array}{l}79 \\
8 \% \\
\end{array}$ \\
\hline & 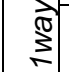 & To & $404 \%$ & $384 \%$ & $20 \%$ & $\begin{array}{l}79 \\
8 \%\end{array}$ \\
\hline & & From & $\begin{array}{l}119 \\
12 \% \\
\end{array}$ & $\begin{array}{l}280 \\
28 \% \\
\end{array}$ & $152 \%$ & \begin{tabular}{|l|l}
$\mathbf{4 1 4}$ \\
$42 \%$ \\
\end{tabular} \\
\hline & 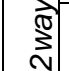 & To & $\begin{array}{l}119 \\
12 \%\end{array}$ & $\begin{array}{l}280 \\
28 \%\end{array}$ & $152 \%$ & $\begin{array}{l}\mathbf{4 1 4} \\
42 \%\end{array}$ \\
\hline \multicolumn{3}{|c|}{ TOTAL } & $\begin{array}{l}303 \\
31 \% \\
\end{array}$ & $\begin{array}{l}636 \\
65 \% \\
\end{array}$ & $475 \%$ & $\begin{array}{l}986 \\
100 \% \\
\end{array}$ \\
\hline \multirow{4}{*}{$\begin{array}{l}w \\
\frac{w}{\delta} \\
\varepsilon \\
d \\
\Sigma\end{array}$} & & From & $333 \%$ & $515 \%$ & $91 \%$ & $\begin{array}{l}93 \\
9 \%\end{array}$ \\
\hline & ্ָত & To & $444 \%$ & $384 \%$ & $111 \%$ & $\begin{array}{l}93 \\
9 \%\end{array}$ \\
\hline & & From & $\begin{array}{l}163 \\
12 \% \\
\end{array}$ & $\begin{array}{l}216 \\
22 \% \\
\end{array}$ & $212 \%$ & $\begin{array}{l}400 \\
41 \% \\
\end{array}$ \\
\hline & $\mid \begin{array}{l}\text { ते } \\
\stackrel{\mathbf{N}}{\mathbf{v}}\end{array}$ & To & $\begin{array}{l}163 \\
12 \% \\
\end{array}$ & $\begin{array}{l}216 \\
22 \% \\
\end{array}$ & $212 \%$ & $\begin{array}{l}\mathbf{4 0 0} \\
41 \% \\
\end{array}$ \\
\hline \multicolumn{3}{|c|}{ TOTAL } & $\begin{array}{l}403 \\
41 \% \\
\end{array}$ & $\begin{array}{l}521 \\
53 \% \\
\end{array}$ & $626 \%$ & $\begin{array}{l}986 \\
100 \%\end{array}$ \\
\hline \multirow{4}{*}{ 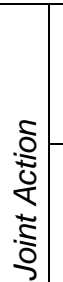 } & & From & $00 \%$ & $00 \%$ & $00 \%$ & $\begin{array}{l}\mathbf{0} \\
0 \% \\
\end{array}$ \\
\hline & 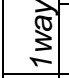 & To & $00 \%$ & $00 \%$ & $00 \%$ & $\begin{array}{l}\mathbf{0} \\
0 \% \\
\end{array}$ \\
\hline & & From & $\begin{array}{l}188 \\
19 \% \\
\end{array}$ & $\begin{array}{l}263 \\
27 \% \\
\end{array}$ & $424 \%$ & \begin{tabular}{|l}
493 \\
$50 \%$ \\
\end{tabular} \\
\hline & $\mid \begin{array}{l}\text { ते } \\
\stackrel{\mathbf{N}}{\mathbf{v}}\end{array}$ & To & $\begin{array}{l}188 \\
19 \% \\
\end{array}$ & $\begin{array}{l}263 \\
27 \% \\
\end{array}$ & $424 \%$ & \begin{tabular}{|l}
493 \\
$50 \%$ \\
\end{tabular} \\
\hline \multicolumn{3}{|c|}{ TOTAL } & $\begin{array}{l}376 \\
38 \%\end{array}$ & $\begin{array}{l}526 \\
53 \% \\
\end{array}$ & $849 \%$ & $\begin{array}{l}986 \\
100 \%\end{array}$ \\
\hline
\end{tabular}

Overall, the reported essential mediation strategies, were distributed differently from strategies currently 
used by researchers (Figure 8). Most frequently reported essential mediation strategies were Reciprocal Joint Action (188), Reciprocal Memories (163) and Reciprocal Expressivity (153).
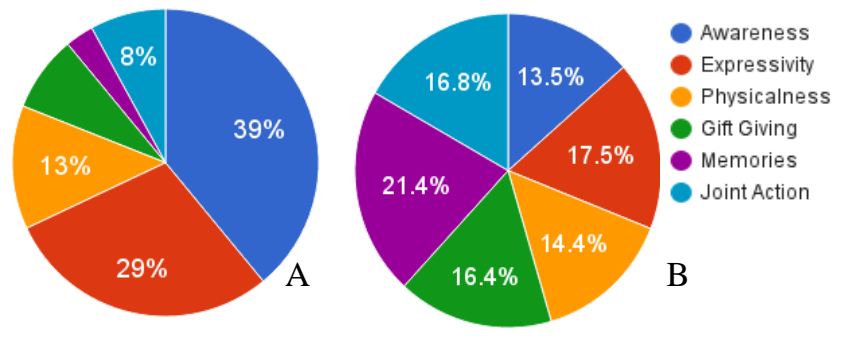

Figure 8: Original Mediation Strategies [presentation of Hassenzahl at al., 2012] (A) \& Reported Essential Mediation Strategies (B)

\section{Q4b: Unwanted Mediation Strategies.}

As with essential strategies, we could anticipate $0 \%$ of unwanted strategies. Instead, near 14\% (805) were identified as "I would not want this" (Figure 9). The most frequently reported unwanted mediation strategies were Reciprocal Awareness (109), Reciprocal Physicalness (97) and Reciprocal Expressivity (66).

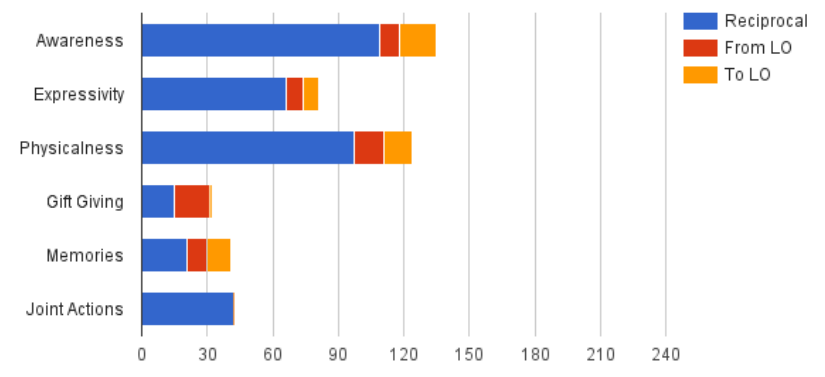

Figure 9: Unwanted Mediation Strategies

\section{Q4c: Ambivalent Mediation Strategies.}

Similarly, we would not anticipate seeing any ambivalence in the preferences for mediation strategies. Yet near half of $(3,145)$ of all possible instances of From and To were identified by participants as ambivalent (Table 7).

\section{Q5: Dyad Preferences of Expanded Mediation Strategies}

Responses for essential, ambivalent and unwanted strategies in Table 7 were aggregated by the types of relational dyads. The contingency table was normalized based on the percentage based on the number of times the original mediation strategy was reported in an individual relational dyad. Percentage numbers were converted into Preference scores. (Table 8).

Based on the medians of the normalized scores, the overall unwanted scores were much smaller than essential scores. Joint Action ( $M=20)$, Reciprocal Memories $(M=18)$ and Reciprocal Expressivity
Table 8: Preference Scores for Expanded Mediation Strategies within specific types of relational dyads (green = top 3 essential for specific dyad, red = top 3 unwanted for specific dyad)

\begin{tabular}{|c|c|c|c|c|c|c|c|c|c|c|c|c|c|}
\hline \multirow{3}{*}{\multicolumn{2}{|c|}{\begin{tabular}{|l|} 
\\
Expanded \\
Mediation \\
Strategies
\end{tabular}}} & \multicolumn{12}{|c|}{$\begin{array}{c}\text { Preference Score of Strategies } \\
\text { by User - Loved one }\end{array}$} \\
\hline & & \multicolumn{2}{|c|}{$\begin{array}{l}\text { Partner } \\
- \\
\text { Partner }\end{array}$} & \multicolumn{2}{|c|}{$\begin{array}{l}\text { Friend } \\
\text { Friend }\end{array}$} & \multicolumn{2}{|c|}{$\begin{array}{l}\text { Sibling- } \\
\text { Sibling }\end{array}$} & \multicolumn{2}{|c|}{\begin{tabular}{|l|} 
Parent- \\
Offsprn \\
$\mathrm{g}$
\end{tabular}} & \multicolumn{2}{|c|}{\begin{tabular}{|l|} 
Offsprin \\
g- \\
Parent
\end{tabular}} & \multicolumn{2}{|c|}{$\begin{array}{l}\text { GrChld- } \\
\text { GrParen } \\
t\end{array}$} \\
\hline & & 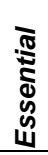 & 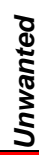 & 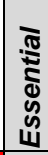 & 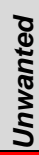 & 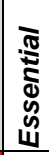 & 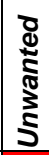 & 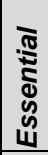 & 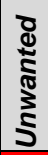 & 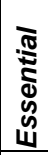 & 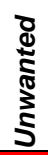 & 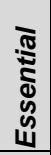 & 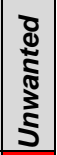 \\
\hline \multirow{3}{*}{ 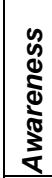 } & Recip & 12 & 4 & 6 & 13 & 4 & 17 & 28 & 7 & 8 & 16 & 2 & 11 \\
\hline & From & 3 & - & 1 & 2 & 4 & - & 4 & - & 2 & 1 & 2 & - \\
\hline & To & 5 & 2 & 2 & 1 & 1 & 2 & 1 & 3 & 3 & 2 & 2 & - \\
\hline \multirow{3}{*}{ 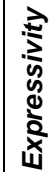 } & Recip & 23 & 1 & 9 & 8 & 13 & 10 & 30 & 4 & 10 & 10 & 5 & 9 \\
\hline & From & 2 & - & 2 & 1 & 1 & 2 & - & 1 & 4 & - & 2 & - \\
\hline & To & 3 & 1 & 1 & 2 & 1 & 1 & 1 & - & 3 & - & 5 & - \\
\hline \multirow{3}{*}{ 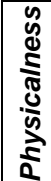 } & Recip & 19 & 2 & 6 & 16 & 5 & 14 & 29 & 6 & 8 & 11 & 7 & 13 \\
\hline & From & 7 & 2 & - & 2 & 1 & 2 & 1 & - & 2 & 1 & 4 & - \\
\hline & To & 2 & - & - & 1 & 2 & 1 & 2 & 1 & 2 & 3 & 4 & 2 \\
\hline \multirow{3}{*}{$\begin{array}{l}0 \\
\vdots \\
0 \\
0 \\
0 \\
0\end{array}$} & Recip & 12 & 1 & 10 & 1 & 6 & 1 & 25 & 1 & 10 & 3 & 9 & 2 \\
\hline & From & 5 & - & 1 & 3 & 2 & 2 & 1 & - & 3 & 3 & 2 & 2 \\
\hline & To & 2 & - & 2 & - & 2 & - & 6 & - & 7 & - & 7 & - \\
\hline \multirow{3}{*}{ @ } & Recip & 15 & 1 & 11 & 3 & 13 & 2 & 33 & - & 15 & 4 & 20 & 2 \\
\hline & From & 4 & - & 3 & 2 & 5 & 1 & - & 1 & 4 & 1 & 2 & - \\
\hline & To & 7 & 1 & 4 & 1 & 2 & 2 & 4 & - & 4 & 1 & 5 & - \\
\hline \multirow{3}{*}{ 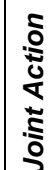 } & Recip & 23 & 3 & 17 & 3 & 18 & 5 & 30 & 1 & 13 & 8 & 16 & 2 \\
\hline & From & - & - & - & - & - & - & - & - & - & - & - & - \\
\hline & To & - & - & - & - & - & - & - & - & - & - & - & - \\
\hline
\end{tabular}

$(M=15)$ were the top three most essential strategies. And Reciprocal Awareness $(M=11)$, Reciprocal Physicalness $(M=10)$ and Reciprocal Expressivity $(M=7)$ were the least wanted strategies.

When looking at essential and unwanted expanded mediation strategies within specific dyads in Table 8, parents (in communication with their offsprings) and romantic partners had on average the highest essential scores for all strategies than any other relational dyad (parent-offspring $M=12$; partners $M=9$; offspring-parent and grandchild-grand-parent $M=6$; friends and siblings $M=5$ ). Both of these dyad categories had low scores for all unwanted strategies (friends, siblings and offspring-parent $M=4$; grandchild-grand-parent $M=3$; parentoffspring $M=2$; partners $M=1$ ).

All strategies in all dyads were both essential and unwanted with a clear difference between the two 
preferences. However, Reciprocal Expressivity was both essential and unwanted between offsprings in communication with parents (10 and 10) siblings (13 and 10) and between.

\subsubsection{Top three essential strategies for:}

Romantic partners found essential Reciprocal Expressivity (23), Joint Action (23) and Reciprocal Physicalness (19). Essential scores for other strategies were high as well with the smallest sore being 12. All unwanted scores were much lower than scores in corresponding essential strategies, with least wanted being Reciprocal Awareness (4) and Joint Action (3).

Friends found essential Joint Action (17), Reciprocal Memories (11) and Reciprocal Gift Giving (10). The top three unwanted strategies were Reciprocal Physicalness (16), Reciprocal Awareness (13) and Reciprocal Expressivity (8).

Siblings found essential Joint Action (18), Reciprocal Expressivity (13) and Reciprocal Memories (13). The top three unwanted strategies were Reciprocal Awareness (17), Reciprocal Physicalness (14), and Reciprocal Expressivity (10).

Parents, who wished to communicate with their offsprings in this way, found essential Reciprocal Memories (33) Reciprocal Expressivity (30) and Joint Action (30). Essential scores for other strategies were high as well with the smallest sore being 25. All unwanted scores were much lower than scores in corresponding essential strategies, with least wanted being Reciprocal Awareness (7) and Reciprocal Physicalness (6).

Offsprings, found essential Reciprocal Memories (15), Joint Action (13), Reciprocal Expressivity (10) and Reciprocal Gift Giving (10). The three top unwanted strategies were Reciprocal Awareness (16), Reciprocal Physicalness (11) and Reciprocal Expressivity (10).

Grandchildren, found essential wished to communicate with their grandparents in this way, were Reciprocal Memories (20), Joint Action (16) and Reciprocal Gift Giving (9). The top three unwanted strategies were Reciprocal Physicalness (13), Reciprocal Awareness (11) and Reciprocal Expressivity (9).

\subsubsection{Types of Desired Augmented Keepsakes}

As part of the open questions within the study, participants were invited to tell us what kind of special keepsake object they might want to use for communication with their loved-one. Participants in 216 dyads choose to share this information with us. Total of 224 artefacts were suggested that naturally fell into four categories: wearable artefacts (93), artefacts that provided physical and / or emotional comfort (50), personalized mementos that could be used or looked at (38), and artefacts that had a particular modality that could be used a memory trigger (43) (Figure 10).

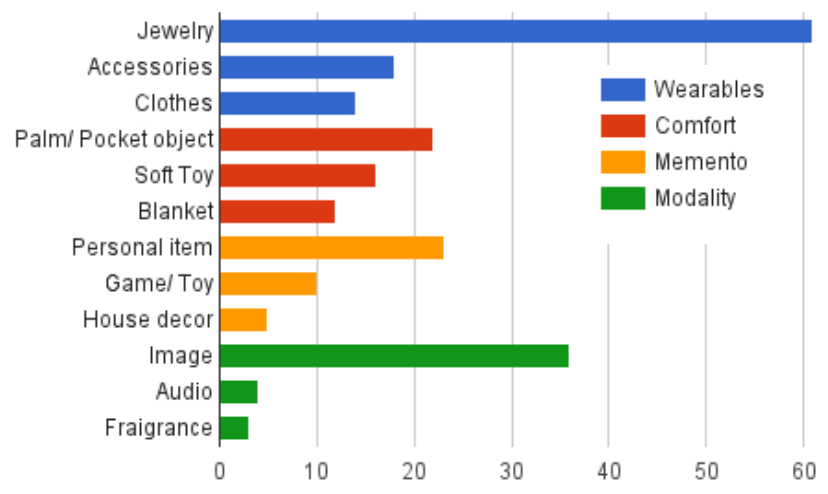

Figure 10: Proposed Augmented Keepsake Artefacts

\section{FINDINGS \& IMPLICATIONS}

Our study was designed to identify the characteristics of potential users of augmented artefacts that would allow for communication with loved-ones without having to write or talk. The study also examined users' preferences within specific types of relational dyads for six strategies for mediation of intimate relationships through technology, commonly used by $\mathrm{HCl}$ researchers (Hassenzahl at al., 2012).

\subsection{User Characteristics}

The present study identified the characteristics of potential users of augmented artefacts that allowed them to communicate with their loved-one without having to call or write. Using semi-structured online questionnaire, the study explored how the likelihood of using augmented artefacts was influences by the type of relationship, the gender of user and loved one, the quality of the relationship, and the frequency of contact with one's loved one. Participants were then asked open-ended questions about the qualities of potential artefacts they might want to use.

\subsubsection{Relationship Type:}

Participants in all types of relational dyads expressed a desire to use these kinds of artefacts; however, romantic partners and offspring were more likely to do so compared to other relational groups. Although parents were the most commonly reported type of relational figures, they were less missed than romantic partners. Interestingly, looking at parentoffspring and offspring-parent dyads, parents appear to be less missed by offspring than the other way around. The asymmetry was further evident in preferences for mediation strategies by both groups. It is possible that this imbalance is a bias resulted from the fact that we did not ask participants to indicate the age of their loved-ones. For example, the offspring that parent-participants referred to 
could be very young, rather than grown-ups. On the other hand, this might be an interesting result that could be explored further to help parents feel more connected with their offspring. Future studies will need to take a closer look at the age of these dyads and the stage of their relationship, and attachment style in addition to relationship quality.

\subsubsection{Gender}

With respect to gender, users in pairs that included at least one female were more likely to want to use augmented artefacts for communication with their loved-ones than male-male pairs. Gender has not been considered in earlier literature, but might be beneficial for selection of relevant user-groups for future research.

\subsubsection{Quality of the relationship}

Users who reported better relationships were more likely to express a desire to use such artefacts. Future relationship-specific studies might find it useful to include measures of attachment security (Fraley, 2010), relationship stage (Knapp, 1978), and relationship quality, such as 'inclusion of other in the self' (Aron et al.,1992). Understanding attachment and psychological closeness in userpairs will enhance our ability to improve users' wellbeing by providing appropriate mediation strategies.

\subsubsection{Frequency of contact}

Romantic partners reported to be in most frequent contact, it was also the most-missed category. In prior research, interaction within this group is generally approached from the perspective of longdistance relationships. However, in our study, participants who reported seeing their loved-one daily or a few times a week, were more inclined to want to use the augmented artefacts, compared to participants who saw their loved one less than once a month. This is consistent with Relationship Psychology which suggest that frequent and small acts of social support within intimate relationships are essential for thriving and wellbeing (Feeney \& Collins, 2014). In this context, our findings provide an additional direction for research in mediation of relationships for loved-ones who see each other frequently.

\subsubsection{Keepsake artefacts}

Participants requested wearable artefacts that could provide emotional comfort, have a special sensory modality, and a special associated memory. Jewelry was the most common form of such artefacts, aligning with a long cultural tradition (Munn, 1993; Scarisbrick \& Fenton, 2013) and creates interesting possibilities with developments in jewelry-like wearable technology (Silina \& Haddadi, 2015).

\subsection{Preferences of Mediation Strategies}

\subsubsection{Reciprocity}

Our study examined six mediation strategies,

currently used by researchers in $\mathrm{HCl}$ (Hassenzahl at al., 2012) from the perspective of the users. The results indicated that although reciprocity is very important, one-way non-reciprocal strategies should also be accounted for. To reflect these, we propose a set of eleven Expanded Mediation Strategies (Table 3). The differences in reciprocity preferences could be due to the attachment styles of users. From the perspective of the Attachment Theory, secure adults are particularly good at the emotional give and take of close relationships. However, secure relationships only account to $60 \%$ of population (Mickelson et al., 1997), leaving $40 \%$ unstudied in the context of Computer Mediated Communication. These reasons could be tested in future targeted dyad-specific studies to help avoid anxiety and unnecessary emotion strain on non-secure users.

\subsubsection{Preferences of strategies}

Only a third of the users indicated that they found certain strategies essential for communication with particular loved-ones; with Joint Action, Reciprocal Memories and Reciprocal Expressivity being the top three most essential. Many indicated that they do not want certain strategies at all; with Reciprocal Awareness, Reciprocal Physicalness and Reciprocal Expressivity being the least wanted. Interestingly, Reciprocal Expressivity was almost equally essential and unwanted by offsprings and by siblings. It is possible that these are due to differences in attachment styles. But it is also possible that there are specific dynamics of communication within these two groups, as they both imply a history of relationships initiated in childhood (Feeney, 2006). It would be interesting to investigate these in the future targeted dyad-specific studies.

Joint Action was one of the top three essential strategies among all dyads. However, in earlier research this was one of the second least employed strategies (Figure 8). This difference could be because Joint Action requires synchronous communication and joint activities that might be difficult to achieve using keepsake-like artefacts. Participants made concrete suggestions of Joint Action artefacts (i.e., games) that could be explored in future research, with consideration to the role of Joint Action in specific types of relationships.

Reciprocal Awareness was one of the top three unwanted strategies by all dyads. However, in earlier research this was the most employed strategy (Figure 8). Awareness requires a high-level of self-disclosure and could also be linked to monitoring and infringing on the privacy of both user and loved-ones. A participant's request for complete awareness and self-disclosure from their partner 
might reflect a preoccupied (insecure) attachment style. In this case, increased Awareness would not lead to increased wellbeing, relationship satisfaction and security. Which is why future studies should take into account the attachment styles and communication needs of both user and partner.

Overall, our findings suggest that users are inclined to prefer different Expanded Mediation Strategies in different types of relationships. The same mediation strategy might be considered essential in one context and decidedly unwanted in another. For example, Reciprocal Physicalness is one of the most essential strategies by partners, but one of the least wanted strategies in other types of relationships. As with earlier described userpreferences, these differences are important considerations for the design of the artefacts that mediate relationships between loved-ones.

\subsection{Limitations}

Our study, had several limitations. Despite using a mixture of closed and open-ended questions, it was mostly reliant on self-reports. In the future, an experimental manipulation of different types of Expanded Mediation Strategies applied to different types of relationships might provide results that are both more robust and specific for each type of dyad. It would be particularly interesting to test and deepen the findings of our study through the use of working artefacts that reflect the suggestions of our participants. It would be beneficial for these future studies to use a standard questionnaire for Measuring Affective Benefits and Costs of Communication Technologies (ABCCT) (Yarosh et al., 2014) to insure consistency between different types of artefacts and to allow researchers to compare future results of different studies.

Even though attachment style is an important underlying concept in communication patterns within the relationships, it was not practical to run a standard attachment questionnaire whilst looking at all the other variables. Despite this limitation, we can predict that people who are less likely to want certain types of mediation are more/less likely to exhibit certain attachment styles. For example, avoidant attachment would probably be associated with unwanted Awareness To, while anxiousambivalent attachment would probably be associated with essential (desired) Awareness From. Future dyad-specific studies on mediation of relationships are needed and should include a measure of attachment, such as standard ECR-R questionnaire (Fraley, Waller \& Brennan, 2000; Fraley, 2010) and a visual measure of the quality of relationship, such as Inclusion of Other in the Self Scale (Aron, Aron \& Smollan,1992).

Similarly, the stage of the relationship is an important factor in communication patterns between loved-ones. For example, partners might want more Expressivity in the early stages of the relationship, and more Memories toward the maintenance stage. Nevertheless, it was not practical to include these variables into our study, and more targeted dyad-specific studies are needed. These should include Knapp's model of Stages of the Relationship (Knapp, 1978), and could be designed to look at the use of mediated artefacts designed for the maintenance of established relationships, or for remedying poor relationships, or advancing new relationships, etc.

\section{CONCLUSION}

Our study contributes to the part of the field of Computer Mediated Communication that is focused on artefacts designed to mediate the sense of relatedness through technology between lovedones. In our paper, we expand this field, by adding perspectives of established theories from related disciplines. Drawing on research and literature from $\mathrm{HCl}$, relationship psychology and communication sciences, the present study identified the characteristics of users and dyads that would want to use such augmented artefacts. These dyads tend to have at least one female, see each other more often than once a month, and report having a good relationship. The study also identified the need to expand currently used six Strategies of Mediating Intimate Relationships though Technology to account for non-reciprocal communication. It further identified that the Expanded Mediation Strategies are not equally essential and indeed not equally wanted. And that these preferences vary for the different types of relational pairs (friends, siblings, offspring-parent, etc.). The paper explored several theoretical and practical implications of the findings, suggesting further studies that will clarify the best uses of augmented artefacts in relationships, with the goal of improving users' wellbeing, reducing negative emotions, and increasing the sense of relatedness between loved-ones.

\section{ACKNOWLEDGEMENTS}

This research is funded by Queen Mary University of London, Media Arts Technology group. We thank all participants, who took time to contribute to this research and shared their preferences and details about themselves and their loved-ones with us.

\section{REFERENCES}

Aron, A., Aron E. N. \& Smollan, D. (1992) Inclusion of Other in the Self Scale \& Structure of Interpersonal Closeness. Journal of Personality \& Social Psychology 63 (45): 596-612.

Bartholomew, K. (1990) Avoidance of Intimacy: An Attachment Perspective. Journal of Social and Personal Relationships. vol. 7 no. 2 147-178. 
Duck, S. (2007) Human Relationships. SAGE Publications Ltd; 4th edition

Feeney, B. C. \& Collins, N. L. (2014) A New Look at Social Support: A Theoretical Perspective on Thriving Through Relationships. Personality and Social Psychology Review.

Feeney, J. A. (2006) Parental attachment and conflict behavior: Implications for offspring's attachment, loneliness, and relationship satisfaction. Personal Relationships. 13 (1): 1134.

Fraley, C. R. (2002) Attachment Stability from Infancy to Adulthood: Meta-Analysis and Dynamic Modeling of Developmental Mechanisms. Personality and Social Psychology Review.

Fraley, C. R. (2010) A Brief Overview of Adult Attachment Theory and Research Background. University of Illinois, 1-8. http://internal.psychology.illinois.edu/ rcfraley/att achment.htm Retrieved 2016-09-19

Fraley, C. R., Waller, N. G. \& Brennan, K. A. (2000) An item-response theory analysis of self-report measures of adult attachment. Journal of Personality and Social Psychology, 78, 350-365.

Hassenzahl, M., Heidecker, S., Eckoldt, K., Diefenbach, S. and Hillmann. U. (2012) All You Need Is Love: Current Strategies of Mediating Intimate Relationships through Technology. ACM Transactions on Computer-Human Interaction, Vol.19, No.4.

Hazan, C. \& Shaver, P. R. (1994) Attachment as an Organizational Framework for Research on Close Relationships. Psychological Inquiry: An International Journal for the Advancement of Psychological Theory 5 (1): 1-22. doi:10.1207/s15327965pli0501_1.
Knapp, M. L. (1978) Social intercourse: from greeting to goodbye. Boston: Allyn and Bacon.

Knapp, M. L. \& Daly, J. A. (2011) The SAGE Handbook of Interpersonal Communication. $4^{\text {th }}$ edition SAGE Publications.

Mickelson, K. D., Kessler, R. C. \& Shaver, P. R. (1997) Journal of Personality and Social Psychology, Vol 73(5), 1092-1106. doi:10.1037/0022-3514.73.5.1092

Munn, G. C. (1993) The Triumph of Love. Thames \& Hudson Ltd

Scarisbrick, D. \& Fenton, J. (2013) Rings: Jewelry of Power, Love and Loyalty. Thames and Hudson Ltd

Sheldon K. M., Elliot, A. J., Kim, Y., \& Kasser, T. (2001). What Is Satisfying About Satisfying Events? Journal of Personality and Social Psychology 80 (2): 325-39.

Silina, Y \& Haddadi, H. (2015) 'New Directions in Jewelry': A Close Look at Emerging Trends \& Developments in Jewelry-like Wearable Devices. In ISWC '15 Proceedings of the 2015 ACM International Symposium on Wearable Computers, 49.

Wright, K. B. \& Webb, L. M. (2010) ComputerMediated Communication in Personal Relationships. Peter Lang Publishing Inc

Yarosh, S., Markopoulos, P., and Abowd. A. D. (2014) Towards a Questionnaire for Measuring Affective Benefits and Costs of Communication Technologies. In: CSCW '14 (ACM) Proceedings of the 17th ACM Conference on Computer Supported Cooperative Work \& Social Computing. ACM, 84-96. 